\title{
Fecal Immunochemical Test (FIT) for Colon Cancer Screening: Variable Performance with Ambient Temperature
}

\author{
Chyke A. Doubeni, MD, MPH, Christopher D. Jensen, PhD, \\ Stacey A. Fedewa, MPH, PhD, Virginia P. Quinn, PhD, Ann G. Zauber, PhD, \\ Joanne E. Schottinger, MD, Douglas A. Corley, MD, PhD, and Theodore R. Levin, MD
}

Introduction: Fecal immunochemical tests (FITs) are widely used in colorectal cancer (CRC) screening, but hemoglobin degradation, due to exposure of the collected sample to high temperatures, could reduce test sensitivity. We examined the relation of ambient temperature exposure with FIT positivity rate and sensitivity.

Methods: This was a retrospective cohort study of patients 50 to 75 years in Kaiser Permanente Northern California's CRC screening program, which began mailing FIT kits annually to screen-eligible members in 2007. Primary outcomes were FIT positivity rate and sensitivity to detect CRC. Predictors were month, season, and daily ambient temperatures of test result dates based on US National Oceanic and Atmospheric Administration data.

Results: Patients $(\mathrm{n}=472,542)$ completed 1,141,162 FITs. Weekly test positivity rate ranged from $2.6 \%$ to $8.0 \%$ (median, $4.4 \%$ ) and varied significantly by month (June/July vs December/January rate ratio $[R R]=0.79,95 \%$ confidence interval $[\mathrm{CI}], 0.76$ to 0.83$)$ and season. FIT sensitivity was lower in June/July (74.5\%; 95\% CI, 72.5 to 76.6) than January/December (78.9\%; 95\% CI, 77.0 to 80.7).

Conclusions: FITs completed during high ambient temperatures had lower positivity rates and lower sensitivity. Changing kit design, specimen transportation practices, or avoiding periods of high ambient temperatures may help optimize FIT performance, but may also increase testing complexity and reduce patient adherence, requiring careful study. (J Am Board Fam Med 2016;29:672-681.)

Keywords: California, Cohort Studies, Colorectal Neoplasms, Early Detection of Cancer, Hemoglobins, Mass Screening, Patient Compliance, Postal Service, Retrospective Studies, Seasons, Temperature, Transportation

Colorectal cancer (CRC) is a leading cause of cancer deaths worldwide. ${ }^{1,2}$ Annual screening using

This article was externally peer reviewed.

Submitted 6 February 2016; revised 19 May 2016; accepted 23 May 2016.

From the University of Pennsylvania (CAD); Division of Research, Kaiser Permanente, Oakland, CA (CDJ, DAC, TRL); American Cancer Society, Atlanta, GA (SAF); Research and Evaluation, Kaiser Permanente, Pasadena, CA (VPQ, JES); Memorial Sloan Kettering Cancer Center, New York, NY (AGZ).

Funding: This study was conducted within the National Cancer Institute-funded Population-based Research Optimizing Screening through Personalized Regimens (PROSPR) consortium, which conducts multisite, coordinated, transdisciplinary research to evaluate and improve cancer-screening processes and supported by a grant from the National Cancer Institute at the United States National Institutes of Health (U54 CA163262).

Conflict of interest: none declared. fecal occult blood testing is recommended and used as an effective approach to reduce CRC incidence and mortality risk. ${ }^{3-6}$ The fecal immunochemical test (FIT) is a newer fecal occult blood test that uses a specific antibody for human hemoglobin. FIT is noninvasive and is more sensitive than traditional guaiac-based tests. ${ }^{7}$ Use of FIT is promoted by a national campaign by the National Colorectal Cancer Roundtable, which includes several primary care organizations, to help increase screening rates for underserved populations. ${ }^{8}$ In population-based screening programs, a FIT kit is often sent to

Corresponding author: Chyke A. Doubeni, MD, MPH, Department of Family Medicine and Community Health, Perelman School of Medicine, University of Pennsylvania, 2 Gates, 3400 Spruce Street, Philadelphia, PA 19104 (chyke.doubeni@uphs.upenn.edu). 
screen-eligible persons to self collect the sample and return in the mail for testing. ${ }^{9}$ This has enabled some programs to screen large numbers of people without the need for an office visit, ${ }^{10}$ a major barrier to CRC screening. ${ }^{11}$

Hemoglobin is unstable at high temperatures and current fecal-based CRC screening tests do not have prominent temperature-related warnings. ${ }^{12,13}$ As experience with FIT accumulates, there is emerging concern that hemoglobin in collected samples may degrade when exposed to high ambient temperatures during transportation, potentially lowering the test's ability to detect preclinical lesions. However, few studies exist on FIT performance characteristics in real-life settings under varying climatic conditions, particularly with mounting concerns about climate change. Only a few prior studies have tried to examine the relationship of ambient temperature exposure with FIT performance. ${ }^{12,13}$ Those studies were relatively small, had limited ability to link FITs to each test's individual level of ambient temperature exposure, and reached conflicting conclusions. To our knowledge, no previous studies have examined the association between ambient temperature and FIT sensitivity.

In this study, we examined the association between exposure to high ambient temperatures at and the positivity rate and sensitivity of FIT in a high-volume population-based CRC screening program in western United States.

\section{Methods}

\section{Study Design and Setting}

This was a population-based retrospective cohort study in CRC screening-eligible patients (defined below) receiving care within Kaiser PermanenteNorthern California's (KPNC) integrated health system. KPNC serves over 3.2 million people in a geographically diverse region whose longitude and latitude spans from $118^{\circ}$ to $124^{\circ}$ west and $36^{\circ}$ to $42^{\circ}$ north, respectively. Except for the San Francisco Bay Area, which usually has mild temperatures year round, the region's populated areas have mild winters and very warm summers. This study was approved by the institutional review boards at both KPNC and the University of Pennsylvania and there was no direct patient contact.

KPNC launched an organized CRC screening program in 2007..$^{9}$ Each year, the program mails
FIT kits (OC FIT-CHEK, Polymedco, Inc.: Cortlandt Manor, NY) to the home addresses of screening-eligible members identified using administrative and electronic medical records data. ${ }^{9}$ During the early years of the program, weekly batches were selected randomly and mailed out during the first 9 to 10 months of the year to allow time for reminders to nonresponders. Therefore, a relatively small number of tests are performed in December and are typically among members who had not responded to outreach earlier in the year. In later years, mailings are timed to the anniversary of the last screening, or on the birthday for patients who were not screened in a prior year. Tests are also given out to patients who are not currently up to date during contacts with health care system such as at flu vaccination clinics.

All FITs performed in KPNC, including kits received at health care office visits or pharmacies are returned by mail to a central receiving facility and then transported to a regional laboratory, both of which are located in the San Francisco Bay Area. Patients are instructed to write the collection date on the kit, and return it by mail as soon as it is collected, but are not instructed to refrigerate the collected samples or avoid hot days when returning the kit for testing. Once transported to the laboratory, specimens are stored according to the manufacturer's instructions in air-conditioned rooms and are tested within 3 days of the receipt date using the automated Polymedco, Inc. OC Sensor DIANA fecal occult blood analyzer system uses rabbit antihuman HbA polyclonal antibodies that are specific for human globin. Specimens are tested within 3 days of arriving at the laboratory, but those collected more than 14 days at the time of testing are not tested or missing collection date are not tested, and patients are sent a new kit to collect a new sample.

\section{Patients}

This study included all KPNC members between January 1, 2007 and December 31, 2011, age 50 to 75 years old on the date of a completed FIT, who were then followed from cohort entry through the end of 2013. Patients were excluded in the calendar year in which they turned 76 years old. We excluded patients who had a prior colectomy and patients diagnosed with CRC were excluded in calendar years following the diagnosis date. Tests received within 10 years of a colonoscopy, within 5 
years of a sigmoidoscopy, or within 1 year of a prior positive fecal occult blood test were also excluded.

\section{Data Sources}

Patients' age, sex, race/ethnicity, medical history, socioeconomic characteristics based on Census data, and residence (defined below) were obtained from electronic databases. Receipt of colonoscopy or sigmoidoscopy was identified using codes from the Current Procedural Terminology and International Classification of Diseases, ninth edition, Clinical Modification.

The FIT result dates were obtained from electronic laboratory databases. We obtained the minimum and maximum daily ambient temperatures and elevation at weather stations from US National Oceanic and Atmospheric Administration (NOAA). We did not have the exact dates patients collected or mailed the specimen, or the date kits were received in the laboratory. Thus, the temperature exposure was assessed by linking each test to 4 dates, at 3 separate locations along a posited sample transportation route: the city of the laboratory on the result date; the city of the central specimen receiving facility on the day before the result date; and at the weather station in the major city of the geographical region where a patient resided on the dates 2 and 3 days before the result date. The average of the measurements at each of these locations was used to define the temperature exposure variables. In cases of multiple weather stations in a region, measurements were weighted using the inverse of the elevation, accounting for potentially lower temperature and population density at higher elevations. The patient's region of residence was based on the KPNC medical center location where she or he received the majority of his or her care and were grouped as Alameda County, San Francisco/Peninsula/San Jose, Walnut Creek/Diablo, North Bay, Central Valley, Fresno, and Sacramento. Tests performed in patients who received their care from contractor services or had missing location information were excluded $(n=4532)$.

We categorized FIT results according to the weeks of each year, months, and seasons. Seasons were categorized as spring, summer, fall, and winter based on equinoxes and solstices. For ease of interpretation, we also combined months with similar average temperatures (December/January, February/March, April/November, May/October, June/July, and August/September).
Weekly temperatures were estimated using time-series methods. Tests were assigned into 5 mutually exclusive groups using the average maximum temperatures aggregated over weekly intervals in each study year. The highest and lowest temperature groups were the weeks with maximum temperatures that were 1.25 S.D. above or below the weekly mean in each year, respectively, and the middle category was the mean \pm 0.25 S.D. We also assessed temperature levels according to deciles in sensitivity analysis.

\section{Outcomes}

The primary outcomes were the FIT positivity rates, defined by a hemoglobin concentration of $100 \mathrm{ng} / \mathrm{mL}$ of buffer (>20 micrograms hemoglobin/gram feces), and diagnosis of CRC that was obtained from cancer registry as has been described previously. ${ }^{14}$ Right-sided (vs left-sided) cancers were defined as those within or proximal to the splenic flexure. FIT sensitivity calculations were based on any CRC diagnosed within 12 months after the test result date. Sensitivity analysis using a 24-month time interval from the result date to cancer diagnosis found similar patterns of results.

\section{Statistical Analysis}

We used time-series techniques to aggregate FIT positivity rates, and the minimum and maximum ambient temperatures, in weekly time periods for each calendar year over the study years. The modified Dickey-Fuller test to assess the statistical significance of observed variations.

Associations between temperature exposure variables and the FIT positivity rate were examined using generalized estimating equations logistic regressions, to account for multiple tests within individuals. We adjusted for age, sex, and race/ethnicity to minimize spectrum bias. We derived rate (relative) ratios (RRs) and 95\% confidence intervals (CI) using predictive margins from the model estimation results.

For analysis of FIT sensitivity, we first used contingency tables to obtain crude FIT sensitivity. To minimize incomplete ascertainment of incident CRC cases in the FIT sensitivity analysis, we only included tests of patients who remained enrolled onto KPNC for at least 12 months after the result date $(\mathrm{n}=1,141,163)$, irrespective of the age at completing FIT. In our analysis, sensitivity was the probability of a positive FIT result given CRC 
diagnosis within 12 months of the test date. Age, sex, and race/ethnicity are associated with both the positivity rate and risk of CRC diagnosis. Thus, we obtained FIT sensitivities adjusted for age and sex according to each temperature exposure variable included in the model (months, seasons, or temperature levels) using generalized estimating equation logistic regressions on all eligible observations by including CRC diagnosis and the temperature indicator variables in the model. We used Bonferonni-adjusted $P$-values (adjusted $\alpha=0.05$ ) to account for multiple comparisons to assess statistical significance of differences. All analyses were generated using STATA version 12.1 (StataCorp LLP, College Station, TX).

\section{Results}

The cohort included 510,922 patients and 1,429,089 eligible FITs over the 7-year study period. A total of 472,542 patients who completed 1,141,163 tests with $\geq 12$ months of followup were used for the primary analyses. The majority of patients were $<65$ years old $(82.1 \%)$, women $(53.9 \%)$, non-Hispanic whites $(57.1 \%)$, and had been in KPNC for 10 years or longer $(60.5 \%$; Table 1$)$.

The temperatures were reasonably normally distributed (Figure 1). On average, the maximum temperatures ranged from $27.6 \pm 8.8^{\circ} \mathrm{C}$ in Fresno to $17.2 \pm 3.5^{\circ} \mathrm{C}$ in the Bay area; the average low temperatures ranged from $7.9 \pm 3.7^{\circ} \mathrm{C}$ in the North Bay to $12.5 \pm 6.3^{\circ} \mathrm{C}$ in Fresno (data are not shown). The hottest months were August and September and the coldest were December and January (Table 2). The daily maximum temperatures based on our 5-level exposure variable ranged from $13.6^{\circ} \mathrm{C}$ (range, 5.4 to 22.7 ) in the lowest temperature group to $23.2^{\circ} \mathrm{C}$ (range, 13.3 to 43.1 ) in the fourth group and $24.4^{\circ} \mathrm{C}$ (range, 15.7 to $37.2^{\circ} \mathrm{C}$ ) in the fifth.

\section{Association of Temperature with FIT Positivity Rate}

The FIT positivity rate decreased with increasing ambient temperatures (Figure 1). In time-series analyses, weekly positivity rate ranged from $2.6 \%$ to $8.0 \%$ (median, $4.4 \%$ ). Significantly higher positivity rates occurred in weeks with lower temperatures, and lower positivity rates occurred in tandem with apparent spikes in ambient temperatures (modified Dickey-Fuller test $P<.01$; Figure 2).
Table 1. Characteristics of the Cohort, Kaiser Permanente-Northern California (KPNC) 2006 to 2013

\begin{tabular}{|c|c|c|}
\hline Characteristics, \% & $\mathrm{N}(472,541)$ & $\%$ \\
\hline \multicolumn{3}{|l|}{ Age, years } \\
\hline 50 to 54 & 204,019 & 43.2 \\
\hline 55 to 59 & 111,756 & 23.7 \\
\hline 60 to 64 & 72,076 & 15.3 \\
\hline 65 to 69 & 52,149 & 11.0 \\
\hline 70 to 74 & 32,541 & 6.9 \\
\hline Females & 254,632 & 53.9 \\
\hline \multicolumn{3}{|l|}{ Race-ethnicity } \\
\hline Non-Hispanic White & 269,639 & 57.1 \\
\hline Non-Hispanic Black & 34,369 & 7.3 \\
\hline Hispanics/Latinos & 57,729 & 12.2 \\
\hline Asian/Pacific Islanders & 68,683 & 14.5 \\
\hline Native Americans & 2,197 & 0.5 \\
\hline Multiple race & 18,959 & 4.0 \\
\hline Unknown & 20,965 & 4.4 \\
\hline \multicolumn{3}{|l|}{ Geographical region } \\
\hline Alameda & 80,225 & 17.0 \\
\hline San Francisco/Peninsula/South Bay & 158,877 & 33.6 \\
\hline North Bay & 46,550 & 9.9 \\
\hline Walnut Creek/Diablo & 47,001 & 9.9 \\
\hline Central Valley & 26,840 & 5.7 \\
\hline Sacramento & 105,783 & 22.4 \\
\hline Fresno & 7,265 & 1.5 \\
\hline \multicolumn{3}{|l|}{$\begin{array}{l}\text { Percentage of households below } \\
\text { federal poverty level, quintiles }\end{array}$} \\
\hline 1 & 100,498 & 21.3 \\
\hline 2 & 98,428 & 20.9 \\
\hline 3 & 96,150 & 20.4 \\
\hline 4 & 92,072 & 19.5 \\
\hline 5 & 84,798 & 18.0 \\
\hline \multicolumn{3}{|l|}{ Charlson Comorbidity Index } \\
\hline 0 & 370,544 & 78.4 \\
\hline 1 & 85,730 & 18.1 \\
\hline 2 & 12,764 & 2.7 \\
\hline $3+$ & 3,503 & 0.7 \\
\hline \multicolumn{3}{|l|}{ Enrollment in health plan, years } \\
\hline 2.0 to 4.99 & 72,675 & 15.4 \\
\hline 5.0 to 9.99 & 114,065 & 24.1 \\
\hline $10.0+$ & 285,801 & 60.5 \\
\hline
\end{tabular}

The positivity rate was highest during the first 2 study years (2007 to 2008) and progressively lower in subsequent years (2009 to 2013, $P$-value for trend $<.01)$. In analyses adjusted for age, sex, and medical region, compared with tests performed in 2007 (positivity rate $=5.0$ ), positivity rates were similar in 2008 (positivity rate $=5.2 ; \mathrm{RR}=1.03$; CI, 0.99 to 1.07$)$. The positivity rate was $4.4 \%$ in $2009(\mathrm{RR}=0.89 ; 95 \% \mathrm{CI}, 0.86$ to 0.93$) ; 4.3 \%$ in 
Figure 1. Distribution of temperatures across the regions over the study period, Kaiser PermanenteNorthern California (KPNC) 2007 to 2013. Note: The horizontal reference line is for the model probability of a positive test $(0.045)$. The temperature distribution in Celsius used the average of the daily minimum and maximums of the entire regions and study period; the average for the 4 temperature categories is: $1,9^{\circ} \mathrm{C} ; 2,11.7^{\circ} \mathrm{C} ; 3,14.3^{\circ} \mathrm{C} ; 4,17.5^{\circ} \mathrm{C}$; and $5,18.7^{\circ} \mathrm{C}$.

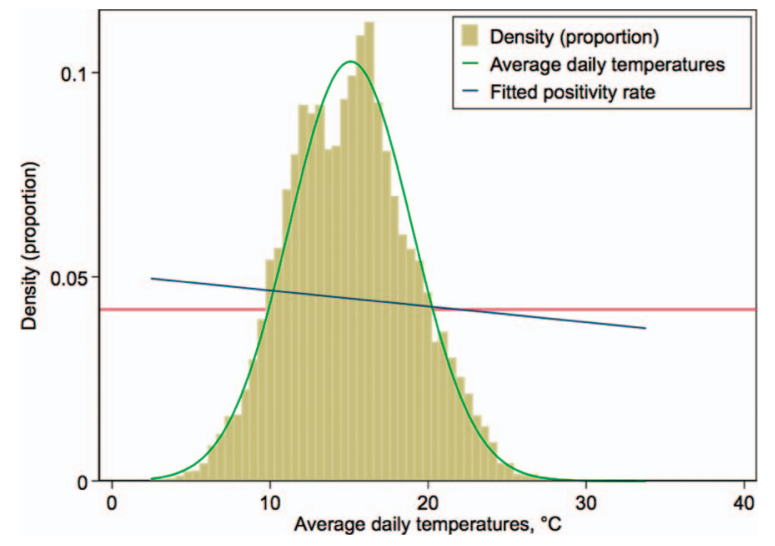

$2010(\mathrm{RR}=0.89 ; 95 \% \mathrm{CI}, 0.85$ to 0.92$) ; 4.3 \%$ in $2011(\mathrm{RR}=0.92 ; 95 \% \mathrm{CI}, 0.88$ to 0.96$) ; 4.0 \%$ in 2012 ( $\mathrm{RR}=0.85 ; 95 \% \mathrm{CI}, 0.85$ to 0.82$)$; and in 2013 , was $3.8 \%$ ( $R R=0.82 ; 95 \% \mathrm{CI}, 0.78$ to 0.85$)$.

In analyses performed according to months over the study period, the positivity rate ranged from $3.9 \%$ during July to $5.8 \%$ during December (Table 2). In regression analyses, FIT positivity varied significantly by months of the year (June/July vs December/January adjusted RR $=0.79 ; 95 \%$ CI, 0.76 to 0.83 ) and by season (summer vs winter: $\mathrm{RR}=0.93$; $95 \%$ CI, 0.90 to 0.96; Figure 3). FIT positivity rates were significantly lower in weeks with higher temperature levels compared with the lower temperature weeks (adjusted $\mathrm{RR}=0.81$; 95\% CI, 0.76 to 0.87 ) (Figure 3, Table 2).

\section{FIT Sensitivity}

A total of 1837 patients had CRC diagnosed within 12 months of a FIT result. The overall sensitivity of FIT for detecting CRC was 75.8\% (95\% CI, 73.8 to 77.8) after adjustment for age, sex, and race/ ethnicity (Table 3) and the unadjusted sensitivity was $77.6 \%$ (95\% CI, 75.6 to 79.5 ) (Supplemental Table 1). In analyses of FIT sensitivity by month, the adjusted sensitivities were highest in January (77.6\%), October (78.0\%), and December (80.7\%) and lowest in April (75.0\%) through July (74.0\%) (Supplemental Table 2). The adjusted sensitivity of FIT was lower for tests completed in June/July (74.5\%; 95\% CI, 72.5 to 76.6) or August/September $(75.6 \%$; $95 \%$ CI, 73.6 to 77.6$)$ than those completed in January/December (78.9\%; 95\% CI, 77.0 to 80.7$)$. Similarly, sensitivity was significantly lower in the summer $(75.0 \%$; $95 \%$ CI, 73.0 to 77.0$)$ than in the winter $(76.4 \%$; $95 \%$ CI, 74.4 to $78.3 \%)$, and autumn $(78.6 \%$; $95 \%$ CI, 76.8 to $80.5 \%$ ) (Bonferonni corrected $P<.01$, Table 3$)$. Sensitivity was lower in the highest temperature $(75.7 \%$; $95 \% \mathrm{CI}$, 73.6 to 77.8$)$ compared with the lowest $(79.6 \%$; $95 \%$ CI, 77.8 to 81.4$)$ temperature weeks. The Bonferonni corrected $P$-values for these comparisons were $<.01$, and was similar for deciles of mean ambient temperatures (Supplemental Table 3).

\section{Discussion}

We used data from a large screening program in a climatically diverse region to evaluate whether levels of ambient temperature were related to the performance characteristics of FIT. Our results showed that significant seasonal variation in the FIT positivity rate and lower sensitivity in hotter months and season with higher temperatures.

Testing for fecal occult blood is the most commonly used method of CRC screening throughout the world..$^{10}$ Depending on whether the tests were performed biennially or annually and whether the tests were or were not rehydrated, use of guaiacbased fecal occult blood test can reduce CRC mortality by $15 \%$ to $33 \%,{ }^{15-17}$ but the efficacy of FIT has not been assessed in randomized trials. ${ }^{3}$ Studies have found greater adherence and acceptability with FIT than with traditional fecal occult blood test because of improved collection devices, fewer required samples, and no dietary restrictions. ${ }^{18,19}$ An added advantage is the ability to use high throughput techniques to enable mass screening. Consistent with our findings, previous estimates of FIT sensitivity for CRC ranged from $65 \%$ to $80 \%$. ${ }^{7,20}$

The performance of a mailed FIT sample is predicated on the ability to preserve globin molecules that may be present in the specimen until it is tested. A Korean study did not find a significant relationship between high ambient temperature exposure on the neoplasia detection rate $^{13}$ and a French study reported no correlation between FIT positivity rate and the season of FIT testing. ${ }^{21} \mathrm{~A}$ 
Table 2. Temperature Ranges and Positivity Rates and Adjusted Rate Ratios for Associations of Daily Ambient Temperatures with Fecal Immunochemical Test Positivity Rate, Kaiser Permanente-Northern California (KPNC) 2007 to 2013

\begin{tabular}{|c|c|c|c|}
\hline Temperature Categories & $\begin{array}{l}\text { Average of the } \\
\text { Minimum-Maximum, }{ }^{\circ} \mathrm{C} \text {, } \\
\text { mean (range) }\end{array}$ & Positive \% & $\begin{array}{c}\text { Positivity Rate Ratio } \\
\text { (95\% Confidence Interval) }\end{array}$ \\
\hline \multicolumn{4}{|l|}{ Temperature levels } \\
\hline 1 (lowest) & $9.6(8.0$ to 11.1$)$ & 5.5 & 1.00 \\
\hline 2 & $11.7(10.3$ to 12.9$)$ & 4.4 & $0.82(0.78$ to 0.86$)$ \\
\hline 3 & $14.3(12.7$ to 15.8$)$ & 4.4 & $0.82(0.77$ to 0.86$)$ \\
\hline 4 & $17.5(15.5$ to 19.4$)$ & 4.2 & $0.78(0.75$ to 0.82$)$ \\
\hline 5 (highest) & 18.7 (16.7 to 20.7$)$ & 4.3 & $0.81(0.76$ to 0.86$)$ \\
\hline \multicolumn{4}{|l|}{ Seasons } \\
\hline Winter & $10.9(9.5$ to 12.4$)$ & 4.5 & 1.00 \\
\hline Spring & $14.8(12.6$ to 16.5$)$ & 4.1 & $0.92(0.90$ to 0.96$)$ \\
\hline Summer & $18.3(16.1$ to 20.1$)$ & 4.2 & $0.93(0.90$ to 0.96$)$ \\
\hline Autumn & $15.4(13.1$ to 17.9$)$ & 5.1 & 1.13 (1.09 to 1.17$)$ \\
\hline \multicolumn{4}{|c|}{ Test result month (individually) } \\
\hline January & $10.0(8.5$ to 11.7$)$ & 4.8 & $0.83(0.77$ to 0.90$)$ \\
\hline February & $11.0(9.8$ to 12.4$)$ & 4.3 & $0.74(0.69$ to 0.79$)$ \\
\hline March & $11.8(10.4$ to 13.0$)$ & 4.3 & $0.75(0.70$ to 0.80$)$ \\
\hline April & $13.5(11.8$ to 14.9$)$ & 4.2 & $0.73(0.68$ to 0.77$)$ \\
\hline May & $15.2(13.5$ to 16.5$)$ & 4.1 & $0.72(0.67$ to 0.77$)$ \\
\hline June & $17.3(15.3$ to 19.0$)$ & 4.1 & $0.72(0.67$ to 0.77$)$ \\
\hline July & $18.1(16.1$ to 19.8$)$ & 3.9 & $0.69(0.64$ to 0.74$)$ \\
\hline August & $18.5(16.2$ to 20.5$)$ & 4.2 & $0.74(0.69$ to 0.79$)$ \\
\hline September & $18.9(16.7$ to 20.9$)$ & 4.4 & $0.77(0.71$ to 0.82$)$ \\
\hline October & $17.2(15.4$ to 18.6$)$ & 4.9 & $0.85(0.79$ to 0.91$)$ \\
\hline November & $13.7(11.8$ to 15.5$)$ & 5.2 & $0.91(0.84$ to 0.98$)$ \\
\hline December & $9.9(8.4$ to 11.5$)$ & 5.8 & 1.00 \\
\hline \multicolumn{4}{|c|}{ Test result month (grouped in pairs) } \\
\hline December/January & $10.0(8.4$ to 11.7$)$ & 5.2 & 1.00 \\
\hline February/March & $11.4(10.2$ to 12.7$)$ & 4.3 & $0.84(0.80$ to 0.88$)$ \\
\hline April/November & $13.6(11.8$ to 15.1$)$ & 4.5 & $0.87(0.83$ to 0.91$)$ \\
\hline May/October & $15.9(14.1$ to 17.4$)$ & 4.4 & $0.86(0.82$ to 0.90$)$ \\
\hline June/July & $17.7(15.7$ to 19.4$)$ & 4.0 & $0.79(0.76$ to 0.83$)$ \\
\hline August/September & $18.6(16.4$ to 20.6$)$ & 4.3 & $0.84(0.80$ to 0.88$)$ \\
\hline
\end{tabular}

Ambient temperature levels were defined using the mean and 1.25 times the standard deviation (SD) of maximum temperatures by year aggregated over weekly intervals, $>$ mean $+1.25 \mathrm{SD},>$ mean, $>$ means- $1.25 \mathrm{SD}$, and $<$ means $+1.25 \mathrm{SD}$ (reference), in that order. Thus, the highest and lowest temperature levels were 1.25 times the level above or below the mean, respectively.

Each set of estimates (by level, month, and by season of the year) was generated individually using generalized estimating equations with logit link and exchangeable covariance matrix, adjusted for age, sex, and medical region. The estimates are relative ratios from the predictive margins.

larger study of 199,654 FITs performed in Florence, Italy, reported $17 \%$ lower probability of a positive result in the summer compared with tests performed in the winter, and the probability of detecting cancer or advanced adenomas was also lower in the summer. ${ }^{12}$ Despite not having exact measures of temperature exposure, we found seasonality and performance variation by temperature exposure levels, suggesting that FIT performance varies significantly at various ambient temperature exposures during transportation. These findings suggest the potential for substantially higher variation in FIT performance in programs using FIT and a need for particular attention to environmental factors to preserve specimen integrity. The effects of temperature may also compound lower programmatic performance from suboptimal adherence. We also observed a statistically significant 
Figure 2. Seasonal variation in the positivity rate of fecal immunochemical test, Kaiser Permanente-Northern California (KPNC) 2007 to 2013. Note: The figure was generated using time series analysis methods for the minimum and maximum air temperatures (min-max temperatures, ${ }^{\circ} \mathbf{C}$ ) and the positivity rates aggregated according to week over the study years. The positivity rate ranged from $2.6 \%$ to $8.0 \%$ ). The false-positive rates paralleled the positivity rate such that the higher the positivity rate, the higher the false-positivity rate.

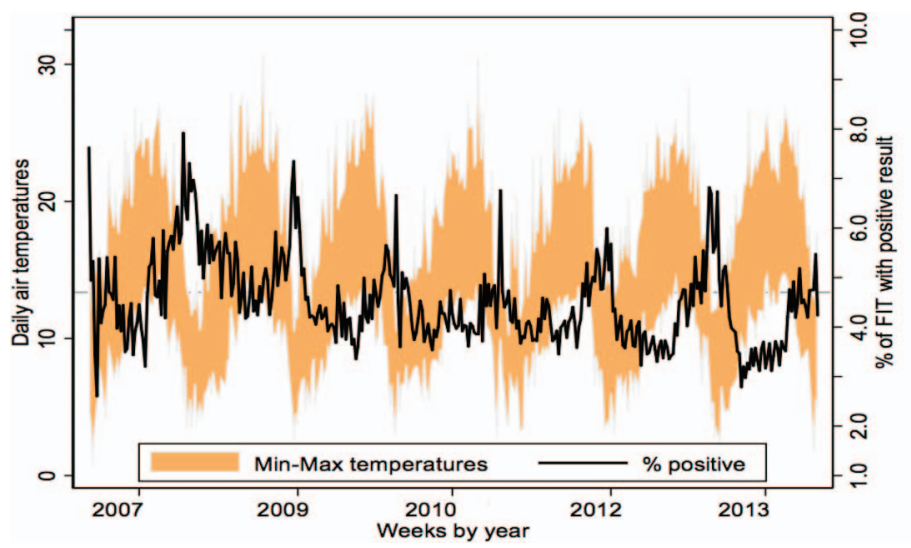

lower positivity rate over the course of the study period. This likely represents the presence of prevalent cancers and thus higher yield during the initial screening round whereas subsequent rounds may be detecting incident cancers.

The variation in weekly positivity rate we observed $(2.6 \%$ to $8.0 \%)$ was large relative to the observed variation in sensitivity of the test. This suggests that the test performs well over a relatively

Figure 3. Association of positivity rate by season, month, and temperature levels, Kaiser Permanente-Northern California (KPNC) 2007 to 2013. *, Each set of estimates (by levels, by month, and by season of the year) was generated, separately, using predictive margins from the logistic regression adjusted for age, race/ethnicity, and sex using the generalized estimating equations approach with exchangeable covariance matrix.

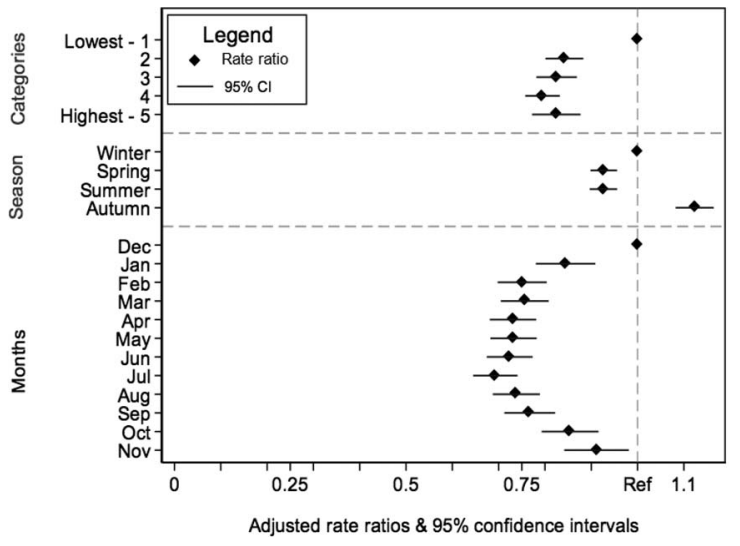

wide range of temperature exposures, although the best performance is achieved during periods of low ambient temperatures such as October through January in our study. However, such optimal time periods may vary depending on climatic conditions in a particular part of the world and over time. Previous studies suggest that hemoglobin may remain in sufficient quantities to be detected even after heat exposure, ${ }^{21,22}$ but may need a lower positivity threshold. This may also account for the relatively small variation in sensitivity, consistent with previous studies suggesting that cancerous lesions produce larger amounts of occult blood than nonmalignant sources of bleeding, ${ }^{23,24}$ but such hypotheses require further study.

This study has some limitations. The effect of ambient temperatures on the FIT sample depends on many factors including storage conditions in mailboxes and postal facilities, direct exposures to high temperatures, and durations of exposures. Specimens stored in a vehicle, for example, may degrade faster because of heat build-up or slower if high temperatures trigger protective processes such as air conditioning. These unmeasured factors may have modified the associations between ambient temperature and test performance, leading to less precise estimates. We did not have exact measurements for level of heat exposure on individual kits during transportation and the length of time samples were exposed to heat (at the home of patients, during transit, and/or at the receiving 


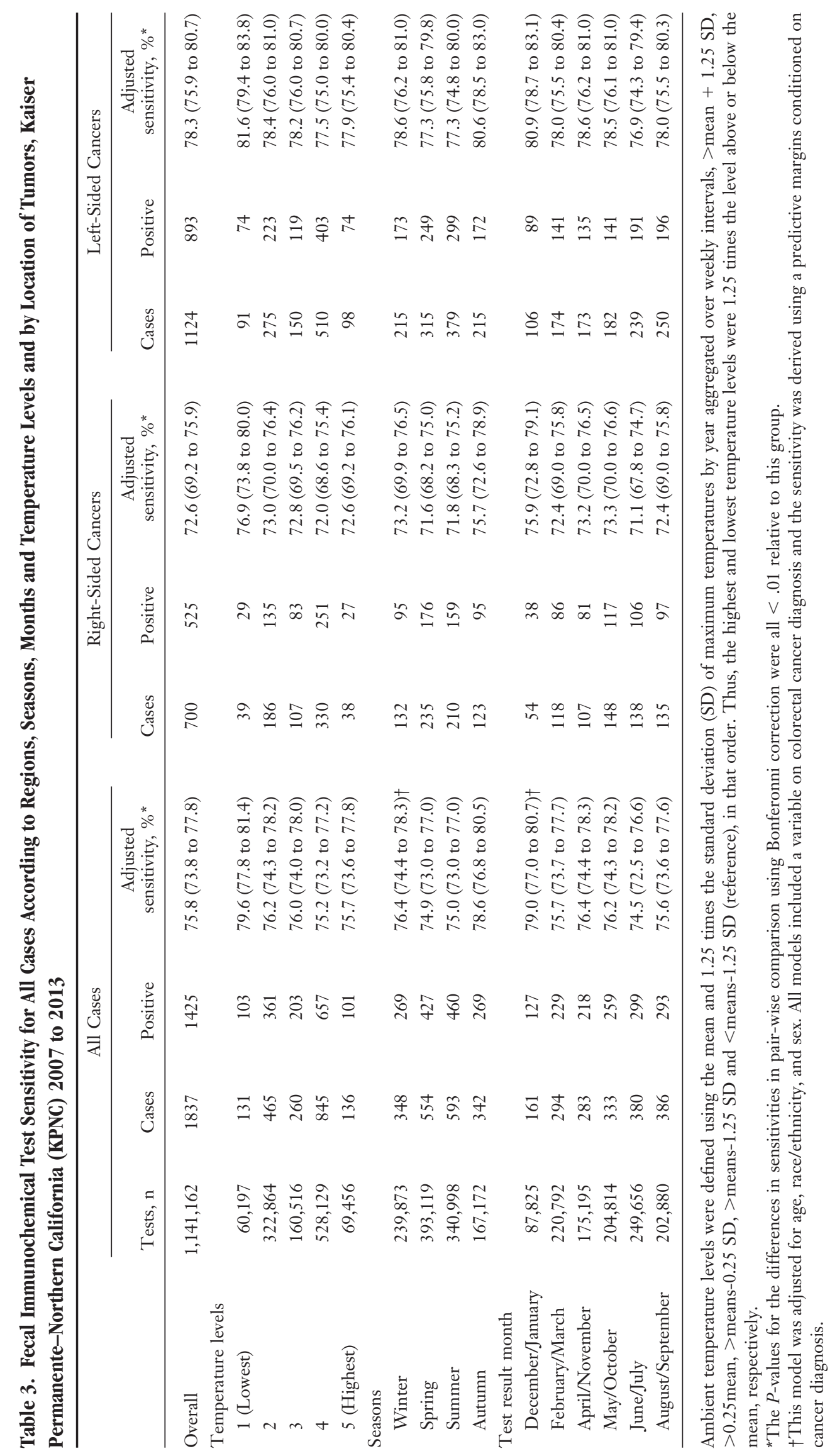


and testing facilities), which are needed to provide precise temperature targets for interventions to optimize FIT performance. We were also unable to account for any potential delays in testing, which may decrease sensitivity. ${ }^{13,22,25}$ Ideally, variation in the yield of FIT should be compared with contemporaneous colonoscopy examinations, which was beyond the scope of this study. We observed that both the positivity rate and sensitivity is lower in months with higher volume of testing. In some analyses, the sensitivity of FIT corresponded with the volume of testing, with warmer periods also experiencing higher testing volumes. However, this correlation was because outreach was done more aggressively during the summer months. Moreover, there is no consistent relationship with testing volume. For instance, the testing volumes, from highest to lowest, were 249,656 in June/July; 220,792 in February/March; 204,814 in May/October; 202,880 in August/September; 175,195 in April/ November; and 87,825 in December/January. Corresponding sensitivities were: $74.5 \%, 75.7 \%$, $76.2 \%, 75.6 \%, 76.4 \%$, and $78.9 \%$, respectively. It is unclear the extent to which potential laboratory delays in testing from higher workload confound results, and deserves further investigation. A small percentage of colonoscopies (1\% or less) are performed outside the health plan and not captured by our electronic ascertainment methods, which will have a minimal effect on our results.

The study has broad generalizability for planning and implementing CRC screening programs in diverse geographic regions around the world. It suggests that steps are needed to enhance the performance of FIT, including potential approaches to help optimize FIT performance during periods, or in regions, of high ambient temperatures while maintaining the ability to reach large numbers of unscreened persons. This may involve an adjustment to the positivity cut point to account for degradation of hemoglobin, avoiding periods of high ambient temperature, changing the kit's design, or modifying specimen transportation practices. The effectiveness of these potential strategies warrants further study.

In conclusion, exposure of FIT samples to high ambient temperatures is associated with reduced FIT positivity rate and sensitivity for detecting CRC. Modifying kit designs and specimen transportation practices, having visible warnings on the kits about sensitivity to heat, and other methods for minimizing high temperature exposures may help optimize FIT performance. However, these measures may also increase testing complexity and reduce patient adherence, thus requiring careful study. Another implication is that, in some regions or seasons, it may be inadvisable to send or receive FIT in the mail if protection from heat exposure cannot be assured. Further research is needed to ascertain ambient temperature levels at which FIT testing may be inadvisable.

\section{References}

1. Siegel R, Naishadham D, Jemal A. Cancer statistics, 2013. CA Cancer J Clin 2013;63:11-30.

2. Ferlay J, Shin HR, Bray F, Forman D, Mathers C, Parkin DM. Estimates of worldwide burden of cancer in 2008: GLOBOCAN 2008. Int J Cancer 2010; 127:2893-917.

3. von Karsa L, Patnick J, Segnan N, et al. European guidelines for quality assurance in colorectal cancer screening and diagnosis: Overview and introduction to the full supplement publication. Endoscopy 2013; 45:51-9.

4. U.S. Preventive Services Task Force. Screening for colorectal cancer: U.S. Preventive Services Task Force recommendation statement. Ann Intern Med 2008;149:627-37.

5. Leddin DJ, Enns R, Hilsden R, et al. Canadian Association of Gastroenterology position statement on screening individuals at average risk for developing colorectal cancer: 2010. Can J Gastroenterol 2010;24:705-14.

6. Zauber AG, Lansdorp-Vogelaar I, Knudsen AB, Wilschut J, van Ballegooijen M, Kuntz KM. Evaluating test strategies for colorectal cancer screening: A decision analysis for the U.S. Preventive Services Task Force. Ann Intern Med 2008;149: 659-69.

7. Allison JE, Sakoda LC, Levin TR, et al. Screening for colorectal neoplasms with new fecal occult blood tests: Update on performance characteristics. J Natl Cancer Inst 2007;99:1462-70.

8. National Colorectal Cancer Roundtable. National Colorectal Cancer Roundtable. 2016. Available from: http://nccrt.org/about/.

9. Levin TR, Jamieson L, Burley DA, Reyes J, Oehrli M, Caldwell C. Organized colorectal cancer screening in integrated health care systems. Epidemiologic Reviews 2011;33:101-10.

10. Benson VS, Patnick J, Davies AK, et al. Colorectal cancer screening: A comparison of 35 initiatives in 17 countries. Int J Canc 2008;122:1357-67.

11. Gupta S, Sussman DA, Doubeni CA, et al. Challenges and possible solutions to colorectal cancer 
screening for the underserved. J Natl Cancer Inst 2014;106:dju032.

12. Grazzini G, Ventura L, Zappa M, et al. Influence of seasonal variations in ambient temperatures on performance of immunochemical faecal occult blood test for colorectal cancer screening: Observational study from the Florence district. Gut 2010;59:1511-5.

13. Cha JM, Lee JI, Joo KR, et al. Performance of the fecal immunochemical test is not decreased by high ambient temperature in the rapid return system. Dig Dis Sci 2012;57:2178-83.

14. Doubeni CA, Field TS, Buist DS, et al. Racial differences in tumor stage and survival for colorectal cancer in an insured population. Cancer 2007;109: 612-20.

15. Mandel JS, Bond JH, Church TR, et al. Reducing mortality from colorectal cancer by screening for fecal occult blood. Minnesota Colon Cancer Control Study. N Engl J Med 1993;328:1365-71.

16. Kronborg O, Jørgensen OD, Fenger C, Rasmussen M. Randomized study of biennial screening with a faecal occult blood test: Results after nine screening rounds. Scand J Gastroenterol 2004;39:846-51.

17. Hardcastle JD, Chamberlain JO, Robinson MH, et al. Randomised controlled trial of faecal-occultblood screening for colorectal cancer. Lancet 1996; 348:1472-7.

18. van Rossum LG, van Rijn AF, Laheij RJ, et al. Random comparison of guaiac and immunochemical fecal occult blood tests for colorectal cancer in a screening population. Gastroenterology 2008;135:82-90.
19. Cole SR, Young GP, Esterman A, Cadd B, Morcom J. A randomised trial of the impact of new faecal haemoglobin test technologies on population participation in screening for colorectal cancer. J Med Screen 2003;10:117-22.

20. Morikawa T, Kato J, Yamaji Y, Wada R, Mitsushima T, Shiratori Y. A comparison of the immunochemical fecal occult blood test and total colonoscopy in the asymptomatic population. Gastroenterology 2005;129:422-8.

21. Dancourt V, Hamza S, Manfredi S, et al. Influence of sample return time and ambient temperature on the performance of an immunochemical faecal occult blood test with a new buffer for colorectal cancer screening. Eur J Cancer Prev 2016;25:109-14.

22. van Rossum LG, van Rijn AF, van Oijen MG, et al. False negative fecal occult blood tests due to delayed sample return in colorectal cancer screening. Int J Cancer 2009;125:746-50.

23. Digby J, Fraser CG, Carey FA, et al. Faecal haemoglobin concentration is related to severity of colorectal neoplasia. J Clin Pathol 2013;66:415-9.

24. McDonald PJ, Digby J, Innes C, et al. Low faecal haemoglobin concentration potentially rules out significant colorectal disease. Colorectal Dis 2013;15: e151-e159.

25. van Roon AH, Hol L, van Vuuren AJ, et al. Are fecal immunochemical test characteristics influenced by sample return time? A population-based colorectal cancer screening trial. Am J Gastroenterol 2012;107: 99-107. 